\title{
Vibration Response Prediction on Rubber Mounts with a Hybrid Approach
}

\author{
Hakan Ucar and Ipek Basdogan \\ Department of Mechanical Engineering, Koç University, Istanbul, 34450, Turkey
}

(Received 12 July 2015; accepted 5 October 2016)

\begin{abstract}
Accurate prediction of the vibration response at a point on a complex structure, where the operational behavior cannot be measured directly, is an important engineering problem for design optimization, component selection and condition monitoring. Identifying the exciting forces acting on the structure is a major step in the vibration response prediction (VRP). At the point where direct measurement is impossible or impractical due to physical constraints, a common approach is to identify the exciting forces based on multiplication of an inverted frequency response function (FRF) matrix and a vector of vibration responses measured at the points where the exciting forces are transmitted through. However, in some cases measuring FRFs are almost impossible. In other cases, where measuring is possible, they may be prone to significant errors. Furthermore, the inverted FRF matrix may be ill-conditioned due to the one or few modes that dominate the dynamics of the structure.

In order to improve the force identification step and reduce the experimental challenges, previous studies focused on either conditioning methods or numerical models. However, conditioning methods result in additional measurements, and using only numerical models causes reduced accuracy due to incongruities between the simulation model and the real system. Considering these problems, a hybrid VRP methodology that incorporates the numerical modeling and experimental measurement results is proposed in this study. Creating an accurate numerical model and properly selecting the force identification points are the main requirements of the proposed methodology. A structure coupled with rubber mounts is used to demonstrate the proposed methodology. The numerical model includes hyperelastic and viscoelastic modeling of the rubber to represent the system behavior accurately. The selection of force identification points is based on a metric that is composed of the average condition number of the FRF matrix across the whole frequency of interest. The results show that the proposed hybrid methodology is superior to other alternative methods where predictions are solely based on numerical results or experimental measurements.
\end{abstract}

\section{INTRODUCTION}

Mechanical systems are usually composed of various subsystems coupled by several links, such as rubber mounts. Any excitation acting on the system is divided into several internal forces, which propagate throughout the mounts. The structural vibration response at a point of interest, as a result of the exciting forces, is usually of great importance in terms of design optimization, component selection and condition monitoring. For the locations in complex structures where the operational behavior cannot be measured directly, a methodology is required for accurate prediction of the response. A major step of the vibration response prediction (VRP) is to identify the exciting forces acting on the structure. The most evident solution is to measure the forces directly. However, this may not be possible due to the complexity of the structure and the challenges of load cell applications. Consequently, indirect methods have been widely studied in the literature. ${ }^{1-20}$ The following studies are worthwhile to mention here. Verheij ${ }^{1}$ introduced the dynamic stiffness method which seems the most straightforward approach, especially for rubber linked structures. However, accurate complex dynamic stiffness data of the rubber mounts is rarely available, and even if present, it is only valid for a given load condition. Another approach, called the transmissibility method, can be implemented to predict the vibration response at a point of interest. ${ }^{2,5}$ In this method, the forces are replaced by the measured responses at the force identification points, and the propagation paths are represented by the transmissibility. This approach is much simpler and faster, but unconsidered potential cross-coupling between the paths can lead to incorrect predictions.

In the early 1980s, the matrix inversion method was developed. ${ }^{6,7}$ The inverse method basically involves multiplication of a vector of vibration responses with an inverted matrix constituted by the frequency response functions (FRFs). The main drawback of this method is the need of the FRF measurements. However, measuring the FRFs is sometimes not possible, especially for complex structures. Even when it is possible, it is very time consuming and prone to significant errors based on excitation, environment, sensor, structure, and unconsidered sources. The possible sources of the above measurement errors are shown in Fig. (1). ${ }^{21-27}$

Modal behavior of the structure also influences the accuracy of the matrix inversion method. The FRF matrix includes information about the vibration modes contributing to the response, and the amplitude of the modal contribution depends on the location of the FRF measurements. Accordingly, the condition number of the FRF matrix, which is simply the ratio of the largest singular value to the smallest, varies significantly across the structure at particular frequencies. In the condition that one or few modes dominate the responses of specific points that constitute the FRF matrix, the rows or columns of the FRF matrix may become linearly dependent resulting in high condition numbers. High condition number refers to smaller singular values. In this case, the solution may not be unique, and the FRF matrix can be defined as "almost singular" or "ill-conditioned". ${ }^{13,14}$ By selecting the points where the forces are identified properly, the condition number can be reduced and ill-conditioning can be improved. Therefore, con- 


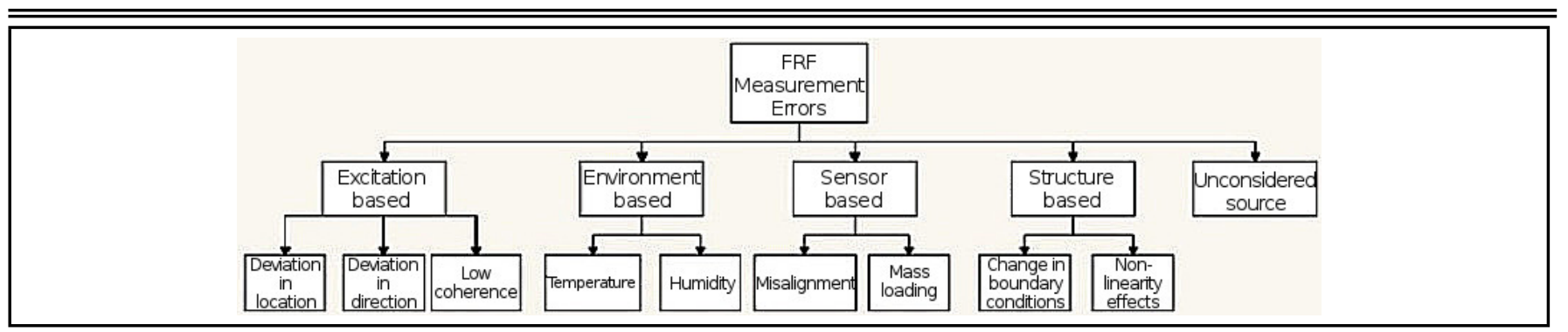

Figure 1. Possible errors in FRF measurements.

dition number of the FRF matrix can be perceived as a good quality indicator of the force identification process.

The ill-conditioned FRF matrix and measurement errors cause erroneous results in the force identification process and consequently, in the response prediction. Thus, eliminating these problems is of great importance in order to improve the accuracy of the prediction results. For this purpose, conditioning methods have been applied in the literature. It has been shown that over-determining the FRF matrix by including additional measurements can improve the inverse problem. ${ }^{12,15}$ However, in this case, the matrix becomes rectangular and a least-squares error method is required for the inversion. In certain cases, the over-determination does not help to reduce the prediction errors, and thus, regularization techniques, such asTikhonov, singular value decomposition, Lcurve etc., are required to make the inverse problem a unique and well-conditioned problem. ${ }^{16-18,28}$ However, these methods are limited to a certain range of condition numbers and sensitive to the selection of the regularization parameters.

As discussed above, previous studies focused on either reducing the experimental measurement efforts and/or improving the conditioning of the FRF matrix. In order to overcome the experimental challenges as well as measurement errors, numerical simulations have been widely used. However, the vibration responses at the force transmission locations required for the inverse method cannot be computed accurately in a numerical model. This is usually due to the fact that the excitation on the structure cannot be modeled precisely.

Conditioning methods result in additional measurements, and using a numerical model causes reduced accuracy due to incongruities between the simulation model and the real system. Considering these challenges, a hybrid VRP methodology that incorporates numerical modeling and experimental measurement results is proposed in this study. In the proposed hybrid methodology, the experimental errors and difficulties of FRF measurements as well as time consumption are eliminated since FRFs are calculated numerically. Moreover, inverse problem is improved by reducing the condition number of the FRF matrix by choosing the right points for the FRF measurements. An accurate numerical model and selection of the points where the forces are identified are critical steps of the proposed methodology. If the representative dynamics of the structure are not included in the measured FRFs due to a wrong selection of the measurement locations, the forces are not identified correctly, and it affects the accuracy of the response prediction process. Accordingly, force identification points are selected based on the "combined condition number" metric within the scope of the hybrid VRP. The proposed hybrid methodology is applied to a structure coupled through rubber mounts. All of the above studies are also validated by applying an experimental VRP study.

This paper is organized as follows: Section 2 focuses on the vibration response prediction methodology based on the direct matrix inversion technique. In Section 3, hybrid VRP method and theoretical considerations such as accuracy of the numerical model and the importance of selection of the force identification points is introduced. Section 4, presents VRP case studies along with the hyperelastic and viscoelastic modeling of the rubber mounts. Finally, the paper is concluded with a summary and conclusion in Section 4.3.

\section{VIBRATION RESPONSE PREDICTION METHODOLOGY}

The vibration response of any point depends on the corresponding transfer function and the force acting on that point if there is only one transmission path. However, if more than one source or path is present, the cross-coupling terms should be taken into consideration. Cross-coupling means that the response at a particular point depends not only on the force acting on that point but also on the other internal forces. ${ }^{29}$ Thus, cross-coupling effects are considered by means of including all FRFs between the transmission paths. Accordingly, vibration response in terms of acceleration can be calculated by multiplying the internal forces and the corresponding FRFs. Assuming that the system is linear and time-invariant, partial contributions from each force are summed up, and the vibration response at the point of interest, $k$, can be predicted, as follows:

$$
\ddot{X}_{k}(\omega)=\sum_{i=1}^{n} \ddot{X}_{k i}(\omega)=\sum_{i=1}^{n} F_{i}(\omega) H_{k i}(\omega) ;
$$

where $n$ is the number of transmission paths.

The major step of the VRP study is to identify the operating forces by implementing indirect methods as discussed above. In the direct matrix inversion method, vibration response measurements are performed at the connection points of the transmission paths, and the FRFs are measured between these points in order to consider the cross-couplings. A square FRF matrix, $n \times n$, is created since the number of forces and responses are equal to each other.Thus, internal forces can be identified in a matrix notation by applying ordinary matrix inversion as shown in Eq. (2). ${ }^{6}$

$$
\left\{F_{j}(\omega)\right\}=\left[H_{i j}(\omega)\right]^{-1}\left\{\ddot{X}_{i}(\omega)\right\}
$$

where $i=j$ which denotes the number of paths and forces, respectively.

\section{HYBRID VIBRATION RESPONSE PREDICTION METHODOLOGY}

In this study, a hybrid VRP methodology was proposed to reduce the measurement effort and errors as well as the ill- 
conditioning of the inverse problem. The hybrid VRP methodology differs from the classical VRP methods in the construction of the FRF matrix. The accelerance (FRF) matrix, $\left[H_{i j}(\omega)\right]$ of Eq. (2), is formed by the numerical FRFs, whereas the acceleration vector, $\left\{\ddot{X}_{i}(\omega)\right\}$ is measured through the experimental studies. For obtaining reliable predictions from the hybrid VRP, an accurate numerical model of the structure needed to be created and validated. Numerical models of the rubber-linked structures are more critical compared to that of rigid structures since rubber may expose the structure to nonlinearity. In this approach, two important steps needed to be completed before performing the hybrid VRP:

a) Numerical FRFs on the structure needed to be validated using measured FRFs.

b) Force identification points needed to be selected properly such that representative dynamics of the structure is included in the measured FRFs. Condition number of the FRF matrix was used as a metric to select the measurement points, and it was related to the spatial position of the measurement points depending on the modal behavior of the structure.

A flow diagram of the hybrid VRP is shown in Fig. (2). The detailed process was as follows;

a) Create a finite element (FE) model of the structure and include all the components.

b) Determine the validation points where the numerical and experimental FRFs are compared. If there are discrepancies between the FRFs over the frequency of interest, the FE model should be updated.

c) After the validation process, select $m$ candidate points to determine the best force identification points.

d) Create a combination with each set of two candidate points.

e) Calculate the coupled FRFs between the points for each combination.

f) Construct the FRF matrix, $\left[H_{i j}(\omega)\right]$ as in Eq. $(2),(2 \times 2)$ for each set of two measurement points $(i, j)$.

g) Calculate the combined condition number, $C_{i j}$ as follows:

$$
C_{i j}=\frac{1}{n}\left(\sum_{\omega} K_{2}\left(\left[H_{i j}(\omega)\right]\right)\right) ;
$$

where $n$ is the number of discrete frequencies and $K_{2}$ stands for the 2-norm condition number.

h) Select the combination having the minimum combined condition number.

i) Measure the vibration responses at the selected points.

j) Calculate the FRFs between the selected and target points from the numerical model.

k) Perform hybrid VRP based on the direct matrix inversion method with the set of experimental measurements and numerical FRFs by implementing Eq. (1) and (2).

\section{VRP CASE STUDIES}

In order to implement the proposed hybrid VRP methodology, an experimental set-up was built as shown in Fig. (3). The set-up consisted of two plates coupled with two rubber mounts.

The schematic representation of the experimental set-up with the force and target locations. T1, T2, and T3, where the responses are predicted, is illustrated in Fig. (4). A modal shaker was fixed to the structure and used to generate the excitations representing the operating source, F. Although there was one operational force acting on the structure, vibrational energy flowed through the rubber links resulting in internal forces. Note thatmoments and rotations were ignored in the calculations since it is very difficult to measure those quantities.

\subsection{Rubber Components Modeling}

Rubber mounts are widely used as vibration isolators as they have elastic and viscous properties such as high inherent damping, deflection capacity, and energy storage. They can also be characterized as compact, easily available, cost effective, and maintenance free. The dynamic properties of the rubber components were of primary concern in designing rubber isolators to reduce transmissibility. In order to determine these properties accurately, hyperelastic and viscoelastic constitutive models were applied. The hyperelastic material model captured the material's nonlinear elasticity with no-time dependence, whereas the viscoelastic model described the material response, which contains an elastic and viscous part depending on time, frequency, and temperature.

The rubber mounts used in the case study were made of natural rubber. In order to evaluate the dynamic properties of the rubber, tensile and relaxation tests were conducted on the specimens created according to DIN 53504-S1 with a thickness of $2 \mathrm{~mm}$, as shown in Fig. (5)a and Fig. (5)b.

\subsubsection{Hyperelastic Constitutive Model}

A hyperelastic material is still an elastic material, which means that the material returns to its original shape once the force is released. The difference is that for a hyperelastic material, the stress-strain relationship derives from a strain energy density function, $W$, as follows: ${ }^{30}$

$$
\sigma=\frac{\partial W}{\partial \varepsilon} .
$$

The strain energy density function can be defined in terms of strain invariants and stretch ratios. Deviatoric and volumetric terms of the strain energy density function for incompressible materials can be written as follwos:

$$
W=W_{d}\left(\bar{I}_{1}, \bar{I}_{2}\right)+W_{v}(J)
$$

where $J$ is the ratio of the final volume to the initial volume and $\bar{I}_{1}, \bar{I}_{2}$ are the strain invariants. Since the rubber was assumed to be incompressible, meaning that the Poisson's ratio is very close to 0.5 , no volumetric test was performed, and the volumetric term was neglected.

Several mathematical models for $W$ were proposed for the analytical and numerical prediction of stress-strain behavior of elastomer materials where the most prominent ones are NeoHookean, Mooney-Rivlin and Yeoh models. ${ }^{31-33}$ All of these 


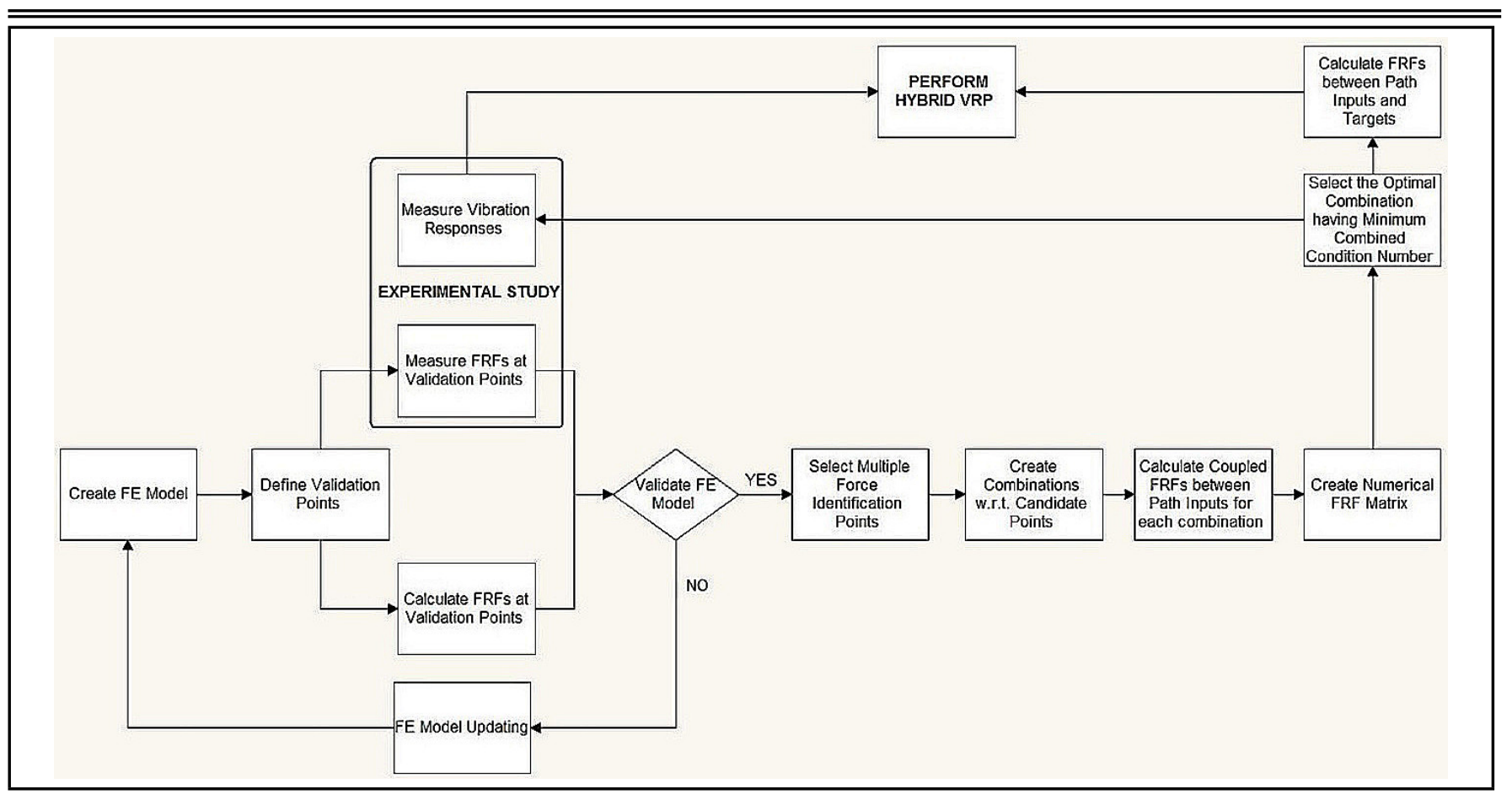

Figure 2. Hybrid VRP flow chart.

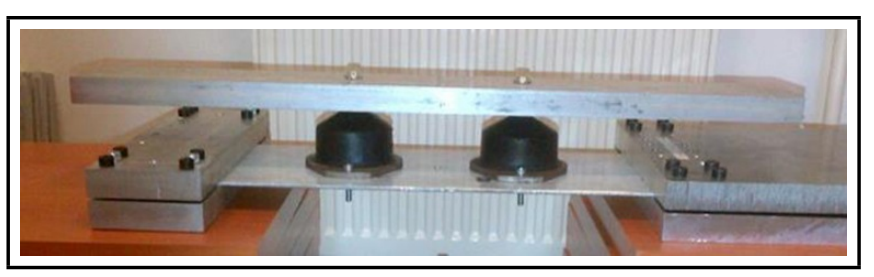

Table 1. Constitutive hyperelastic material models.

\begin{tabular}{||c|c|}
\hline Hyperelastic Model & Strain Energy Density Function \\
\hline Neo-Hookean & $W=C_{10}\left(I_{1}-3\right)$ \\
\hline Mooney-Rivlin & $W=C_{10}\left(I_{1}-3\right)+C_{01}\left(I_{2}-3\right)$ \\
\hline Yeoh & $W=C_{10}\left(I_{1}-3\right)+C_{20}\left(I_{1}-3\right)^{2}$ \\
& $+C_{30}\left(I_{1}-3\right)^{3}$ \\
\hline
\end{tabular}

Figure 3. The experimental setup coupled with rubber mounts.

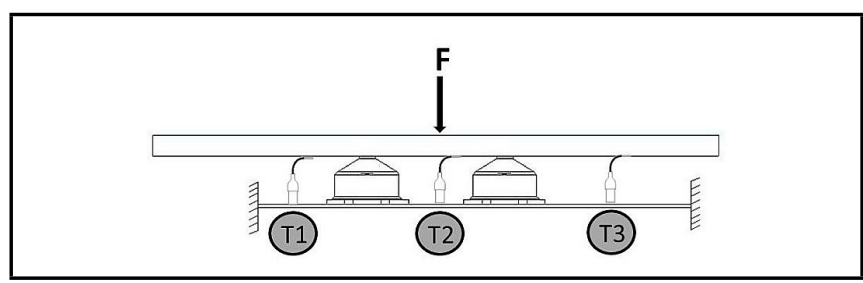

Figure 4. Schematic representation of the set-up.

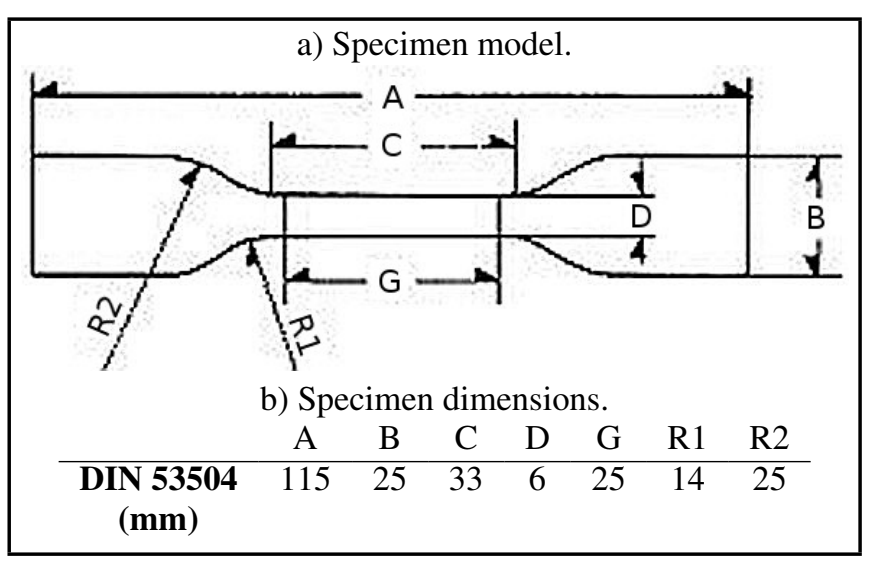

Figure 5. Rubber specimen.

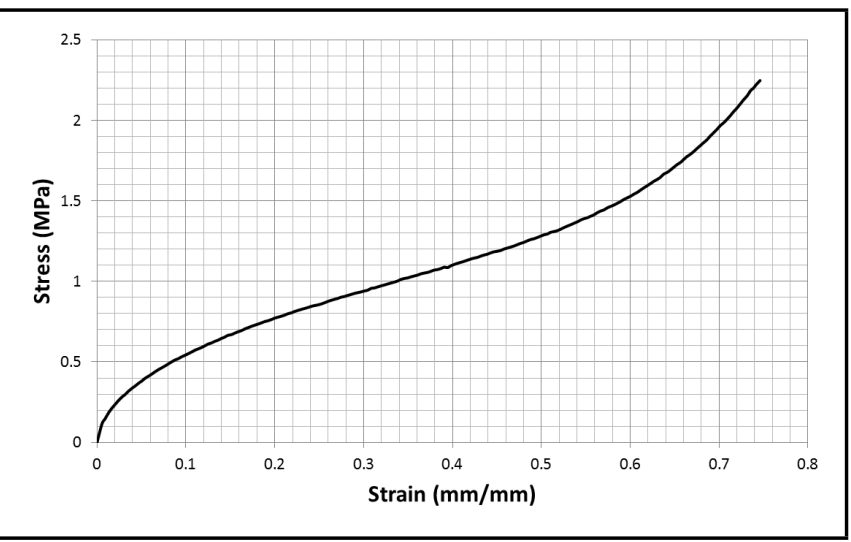

Figure 6. Stress-strain responses from uniaxial tensile test.

models are semi-empirical models and require experimental parameters from shear, uniaxial, or biaxial tests. In this study, Neo-Hookean, Mooney-Rivlin and Yeoh models were considered and the corresponding mathematical models are shown in Table 1.

To study the hyperelastic properties, specimens were subjected to quasi static tensile loading with constant strain rate of $25 \mathrm{~mm} / \mathrm{min}$. Three cycles of the experiment were conducted to minimize experimental errors. Figure (6) presents the stressstrain responses obtained from the quasi static test, and the estimated coefficients of hyperelastic models for the rubber component were tabulated in Table 2. 
Table 2. Estimated hyperelastic coefficients of the rubber.

\begin{tabular}{|c|c|c|c|c|}
\hline $\begin{array}{c}\text { Hyperelastic } \\
\text { Model }\end{array}$ & $C_{10}$ & $C_{20}$ & $C_{30}$ & $C_{01}$ \\
\hline Neo-Hookean & 0.6933 & - & - & - \\
\hline Mooney-Rivlin & 0.3724 & - & - & 0.4397 \\
\hline Yeoh & 0.9636 & -06213 & 0.3265 & - \\
\hline
\end{tabular}

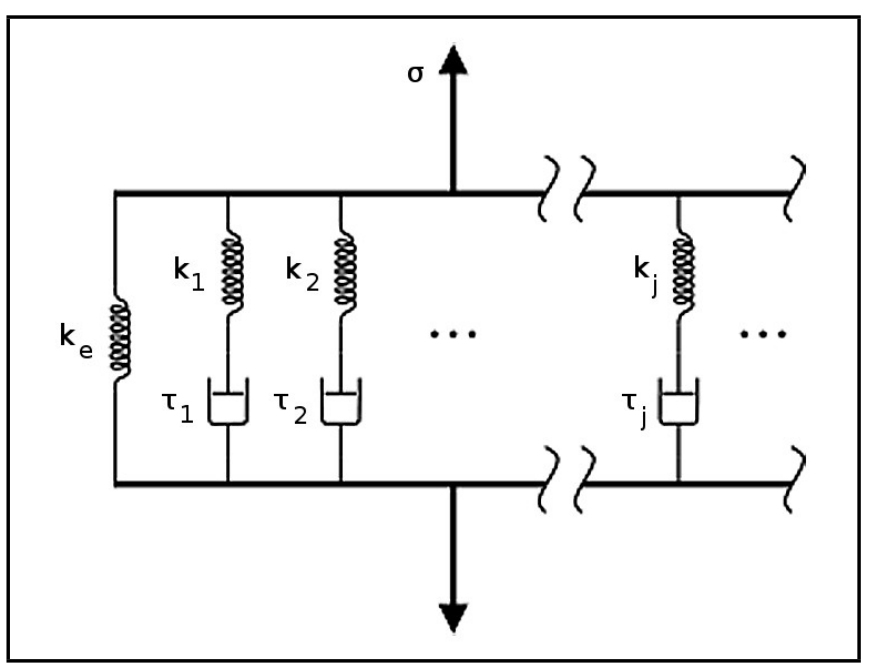

Figure 7. Generalized Maxwell model. ${ }^{39}$

\subsubsection{Viscoelastic Rheological Model}

As discussed before, the hyperelastic model represents the nonlinear elastic response with no time dependence. In order to model time dependency of the rubber behavior, a viscoelastic rheological model was used by means of a relaxation test. In a relaxation test, the strain is held constant and the stress decreases with time. ${ }^{34-37}$ One of the basic rheological viscoelastic models is the Maxwell model, which includes both the elastic and viscous property of the material. The Maxwell model consists of a linear ideally viscous, Newtonian dashpot and linear elastic Hookean spring in series. In the advanced form of this model, which is called the generalized Maxwell model, the relaxation does not take place at a single time but during a series of times. Thus, it can have many spring-dashpot Maxwell elements as shown in Fig. (7). ${ }^{38}$ Thismodel can anticipate relaxation behavior and is usually applied in the case of small deformations. ${ }^{35,36}$

Prony series is one of the best functions for modeling the linear viscoelasticity and the generalized Maxwell model. The resulting stress vs. time data from a tensile relaxation test can be fitted with Prony series as shown in Eq. (6).

$$
E(t)=E_{\infty}+\sum_{i=1}^{N} E_{i} \exp \left(-\frac{t}{\tau_{i}}\right)
$$

where $E_{\infty}$ is the long-term modulus, $\tau_{i}$ is the relaxation time and $N$ is a finite integer. The instantaneous modulus is given by $E(t=0)$.

The relaxation behavior of the rubber specimens was examined through relaxation tests. Figure (8) shows the time history of the stress decrement at a constant strain. The relaxation curve initially revealed a very fast stress relaxation and then, slowed down and continued in an asymptotic sense as confirmed in the references. ${ }^{40,41}$ The generalized Maxwell model was used for the rubber components used in this study, and the Prony coefficients were determined by applying curve fitting to the relaxation test data. These coefficients were used for mod-

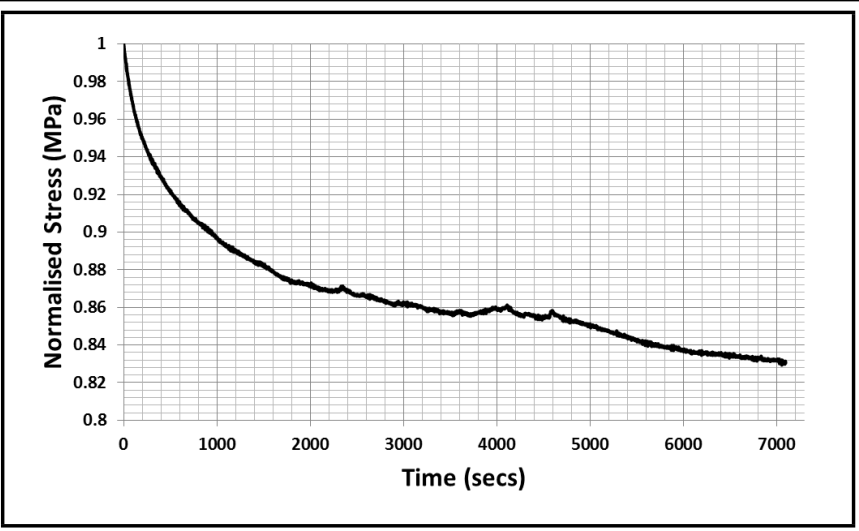

Figure 8. Normalized relaxation test results for the rubber specimen.

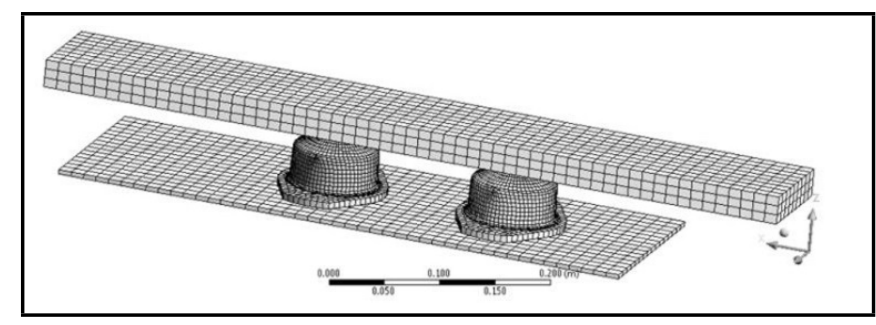

Figure 9. Numerical FEM model of the experimental set-up.

eling the viscoelastic properties of the rubber in the FE model, which is presented in the following section.

\subsection{Numerical Model of the Set-up and Validation}

As discussed in previous sections, an accurate numerical model was required in order to implement hybrid VRP study. For this purpose, the measured properties of the rubber, presented in previous section, was used for modeling the rubber mounts, and the numerical model of the set-up was generated by the finite element method (FEM) tool, ANSYS, as shown in Fig. (9).

A system with rubber components can display nonlinearity due to the hyperelastic properties of the rubber. The equation of motion of a general nonlinear system subjected to a time dependent excitation is as stated in Eq. (7).

$$
\begin{aligned}
{[M]\{x(t)\}+[C]\{\dot{x}(t)\} } & +i[D]\{x(t)\}+[K]\{x(t)\} \\
& +\{G(\dot{x}(t), x(t)\}=\{f(t)\}
\end{aligned}
$$

where $M, C, D$, and $K$ are mass, viscous damping, hysteretic damping, and stiffness matrices, respectively. The nonlinear component of the system is represented by the nonlinear vector, $G$, which is a function of all displacements and velocities in a general case. Due to the nonlinear material properties of the model, linear harmonic analysis could not be implemented in FEM solvers. Thus, transient analysis was used for a more accurate solution. ${ }^{42}$ The responses obtained in the time domain were transformed to the frequency domain using post processing and the Fast Fourier Transform (FFT) algorithm in MATLAB, as described in Fig. (10). In the algorithm, time response data was divided into smaller blocks in order to compute multiple FFTs instead of computing a single FFT for the whole data set. Overlapping, windowing, and averaging was used in order to obtain a good representation of the data set and improve the accuracy of FFT. 


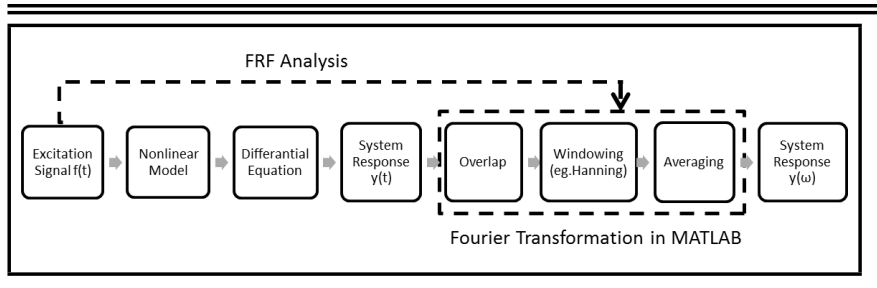

Figure 10. Numerical simulation algorithm.

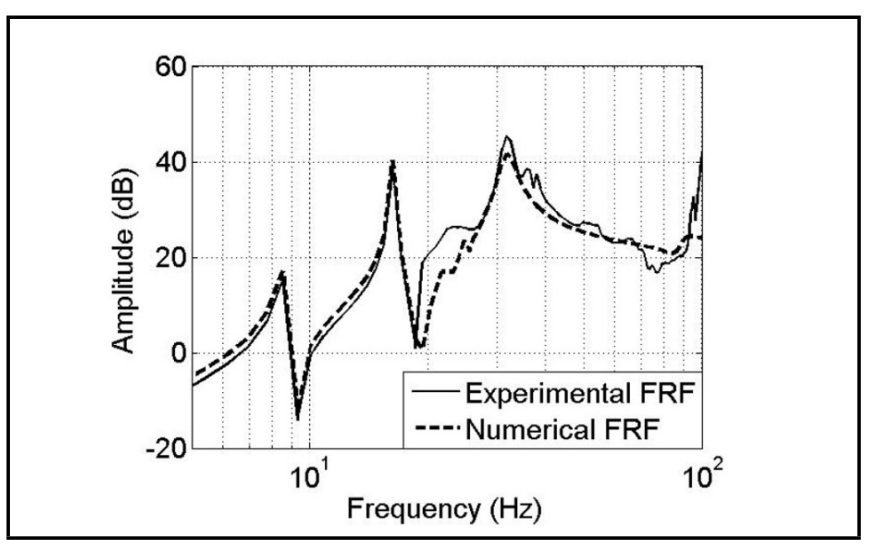

Figure 11. A representative FRF of the experimental set-up.

The first step of the hybrid VRP was the model validation, which was performed by comparing the numerical FRFs with the measured ones. A representative FRF is shown in Fig. (11), and it can be seen that the numerical FRF agrees reasonably well with the experimentally measured FRF. Other selected points were also verified to assure that the numerical model could be accepted as valid.

\subsection{Selection of the Force Identification Points}

Another consideration for the hybrid VRP was the condition number of the accelerance matrix. Thus, numerical simulations were carried out to select the points where the transmitted force would be identified. The points were selected based on the "combined condition number" metric as discussed in Section 3. As shown in Fig. (12), a total of 17 candidatepoints were selected and the accelerance matrix, $\left[H_{i j}(\omega)\right]$, was created for each set of 2 candidate points. A total of 136 combinations were considered. Combined condition numbers for each combination were shown in Fig. (13). According to Fig. (13), out of 136 combinations, the best and worst combinations are determined as 5-8 and 13-16, respectively. The condition numbers of the accelerance matrices formed by these combinations are given in Fig. (14) as a function of frequency. As shown in the figure, the condition numbers were reduced considerably when the accelerance matrix was created by the best combination.

\subsection{Experimental and Hybrid ORP Results}

After selecting the measurement points, vibration responses of each target on the structure were predicted by implementing Eq. (1) and are shown in Fig. (4). The responses of T1 and T2 points predicted by the hybrid VRP with the best and worst combinations were compared with the experimentally measured ones in Figs. (15)a and (15)b. All of these results were also compared with the responses predicted by the experimental VRP where all required operational responses and

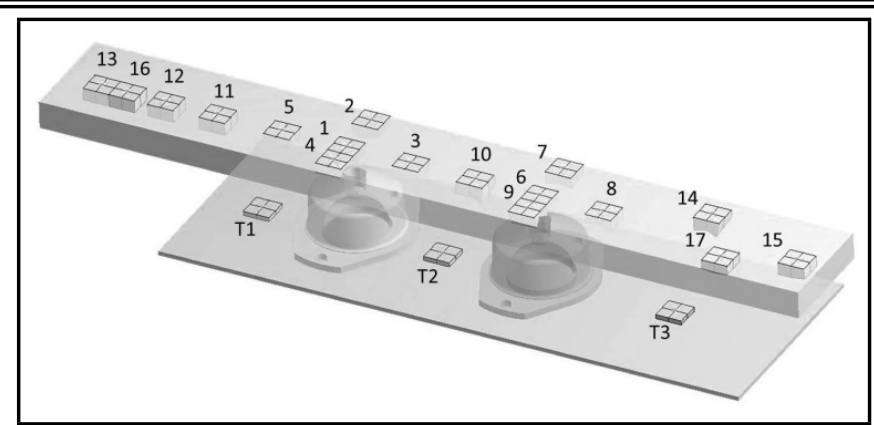

Figure 12. Candidate force identification points.

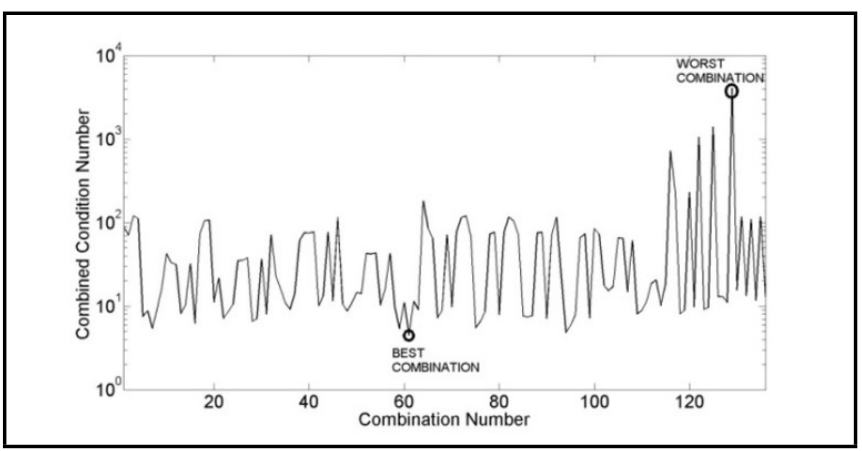

Figure 13. Combined condition number for each combination of two points.

FRFs were obtained experimentally for validation purposes. The condition number of the numerical accelerance matrix was considerably lower than that of the experimental accelerance matrix and is shown in Fig. (16). Due to the reduction in the condition numbers, it clearly shows that the hybrid VRP method with the best combination improves the results significantly. Note that, a similar behavior was exhibited by the other measurement location, T3. It can be seen that the combined condition number is an efficient tool for selecting the force identification points. However, closer inspection reveals some discrepancies at specific frequencies for both methods. These discrepancies may have occurred due to the unconsidered inplane lateral forces, rotations, and moments.

\section{CONCLUSIONS}

A hybrid vibration response prediction methodology was proposed for rubber linked structures with numerical and experimental case studies. FRF measurements were obtainednumerically and vibration responses were measured experimentally in the proposed method. A validated numerical model of the structure which incorporates hyperelastic and viscoelastic behavior of rubber mounts, was used for the hybrid VRP method. It was demonstrated that the selection of the force

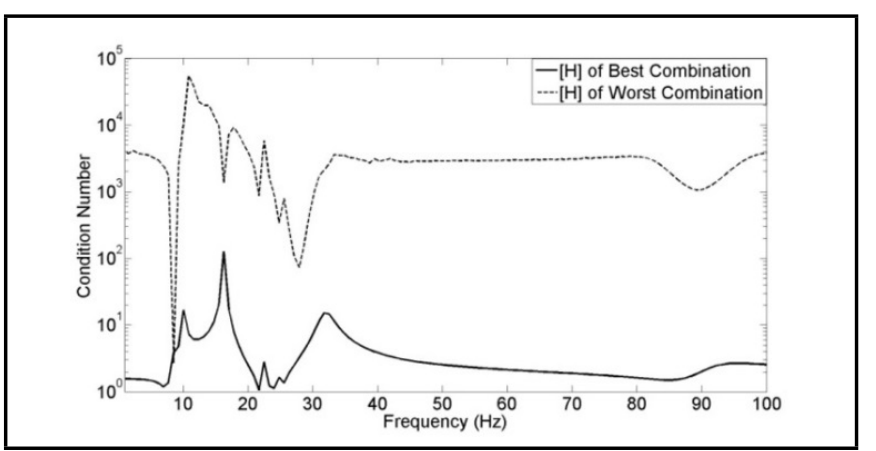

Figure 14. Condition numbers of best and worst combinations. 


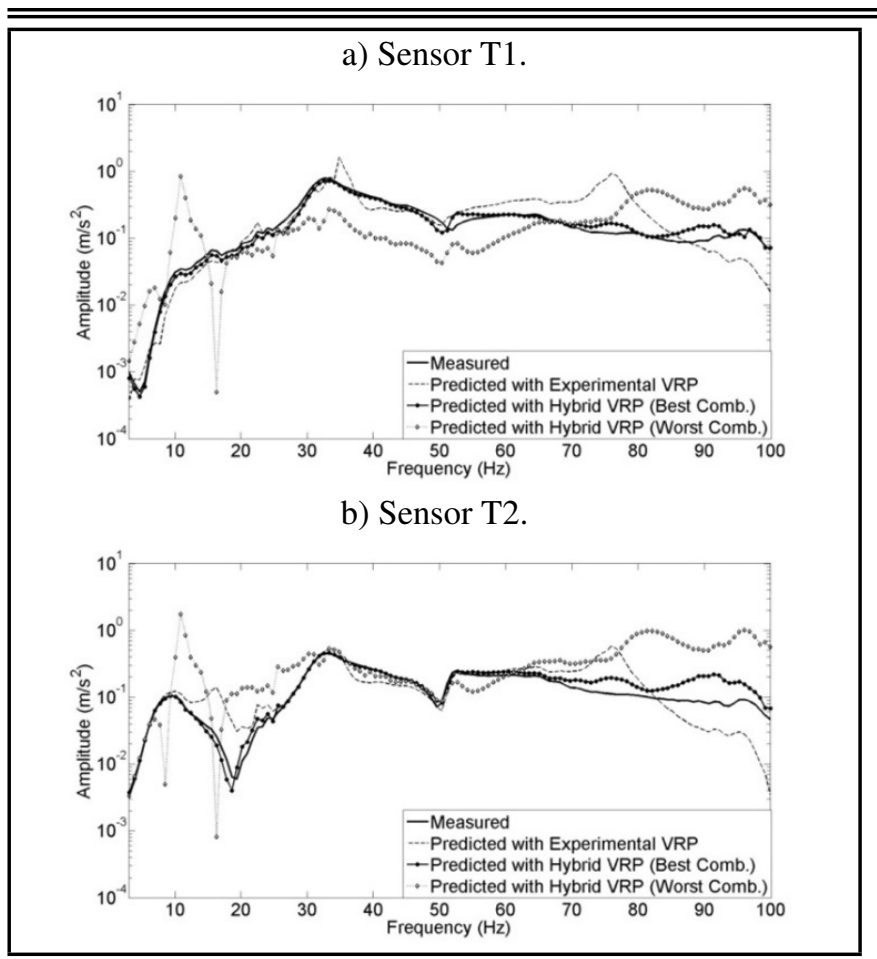

Figure 15. Predicted acceleration responses.

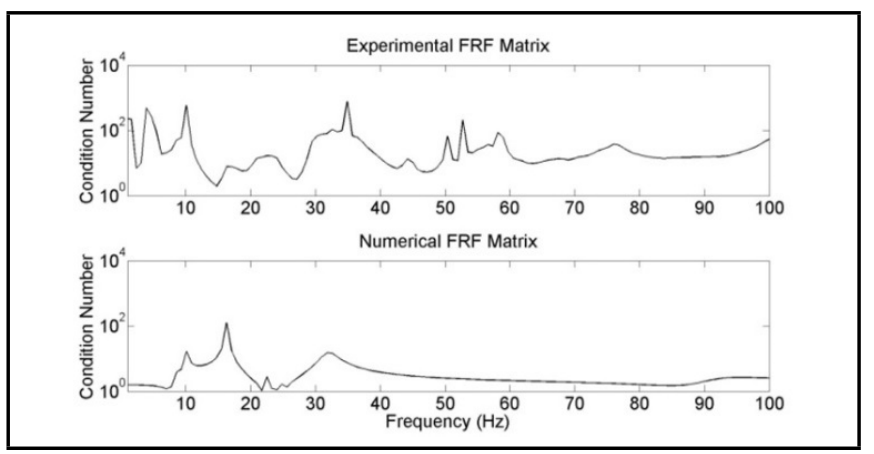

Figure 16. Condition numbers of experimental and numerical accelerance matrices.

identification points plays an important role in determining the condition number of the accelerance matrix. The force identification point selection is based on a metric that is composed of the average condition number of the FRF matrix across the whole frequency of interest. The results show that the proposed hybrid methodology is superior to other alternative methods where predictions are solely based on the numerical results or experimental measurements. Consequently, the hybrid VRP methodology can be considered a reliable and efficient tool to predict the vibration responses, especially for complex structures.

\section{ACKNOWLEDGEMENTS}

The authors would like to thank Tekno Kaucuk Sanayi AS for their support on test plans, test equipment, and data collection.

\section{REFERENCES}

1 Verheij, J. W. Multipath Sound Transfer from Resiliently Mounted Shipboard Machinery, TNO, Delft, Netherlands, (1982).
2 Magrans, F. X. Method of measuring transmission paths, Journal of Sound and Vibration, 74 (3), 321-330, (1981). https://dx.doi.org/10.1016/0022-460X(81)90302-3

3 Ribeiro, A. M. R., Silva, J. M. M., and Maia, N. M. M. On the generalisation of the transmissibility concept, Mechanical Systems and Signal Processing, 14 (1), 29-35, (2000). https://dx.doi.org/10.1006/mssp.1999.1268

4 Maia, N. M. M., Silva, J. M. M., and Ribeiro, A. M. R. The transmissibility concept in multidegree-of-freedom systems, Mechanical Systems and Signal Processing, 15 (1), 129-137, (2001). https://dx.doi.org/10.1006/mssp.2000.1356

5 Ribeiro, A. M. R., Fontul, M., Maia, N. M. M., and Silva, J. M. M. Further developments on the transmissibility concept for multiple degree of freedom systems, Proceedings of the XIth International Conference on Vibration Engineering, Timisoara, Romania, (2005).

${ }^{6}$ Desanghere, G. Identification of external forces based on frequency response measurements, Proc. 8th Int. Seminar on Modal Analysis, Part 3b, Leuven, Belgium, (1983).

7 Roesems, D. Analysis of structure borne noise transmission paths, Proc. 6th Int. Seminar on Modal Analysis, Leuven, Belgium, (1981).

8 Verheij, J. W. Experimental procedures for quantifying sound paths to the interior of road vehicles, Proc. 2 nd Int. Conference on Vehicle Comfort, 483-491, Bologna, Italy, (1992).

9 Starkey, J. and Merril, G. On the ill-conditioned nature of indirect force measurement techniques, Int. Journal of Analytical Experimental Modal Analysis, 4, 6, (1989).

10 Freymann, R. and Stryczek, R. Noise path analysis and optimization, Proc. Unikeller Conference, Zurich, Switzerland, (1995).

11 Van der Linden, P. J. and Floetke, H. Comparing inverse force identification and the mount stiffness force identification methods for noise contribution analysis, Proc. International Conference on Modal Analysis Noise and Vibration Engineering, 2971-2986, Leuven, Belgium, (2004).

12 Mas, P., Sas, P., and Wyckaert, K. Indirect force identification based upon impedance matrix inversion: a study on statistical and deterministical accuracy, Proc. 19th Int. Seminar on Modal Analysis, Leuven, Belgium, (1994).

13 Fabunmi, J. A. Effects of structural modes on vibratory force determination by the pseudoinverse technique, AIAA Journal, 24 (3), 504-509, (1986). https://dx.doi.org/10.2514/3.9297

14 Thite, A. N. and Thompson, D. J. Selection of response measurement locations to improve inverse force determination, Applied Acoustics, 67 (8), 797-818, (2006). https://dx.doi.org/10.1016/j.apacoust.2006.01.001

15 Hendrickx, W. Accurate vehicle FRF measurements for indirect force determination based upon matrix inversion, Proc. 19th Int. Seminar on Modal Analysis, 1037-1048, Leuven, Belgium, (1994). 
16 Thite, A. N. and Thompson, D. J. The quantification of structure-borne transmission paths by inverse methods. Part 1: Improved singular value rejection methods, Journal of Sound and Vibration, 264 (2), 411-431, (2003). https://dx.doi.org/10.1016/s0022-460x(02)01202-6

17 Thite, A. N. and Thompson, D. J. The quantification of structure-borne transmission paths by inverse methods. Part 2: Use of regularization techniques, Journal of Sound and Vibration, 264 (2), 433-451, (2003). https://dx.doi.org/10.1016/s0022-460x(02)01203-8

18 Choi, H. G., Thite, A. N., and Thompson, D. J. A threshold for the use of Tikhonov regularization in inverse force determination, Applied Acoustics, 67 (7), 700-719, (2006). https://dx.doi.org/10.1016/j.apacoust.2005.11.003

19 Verheij, J. W. Inverse and reciprocity methods for machinery noise source characterization and sound path quantification. Part 2: transmission paths, Int. Journal of Acoustics and Vibration, 2 (3), 10, (1997). https://dx.doi.org/10.20855/ijav.1997.2.313

20 Verheij, J. W. Inverse and reciprocity methods for machinery noise source characterization and sound path quantification. Part 1: Sources, Int. Journal of Acoustics and Vibration, 2 (1), 10, (1997). https://dx.doi.org/10.20855/ijav.1997.2.107

21 Biermayer, W., Brandl, F., Höldrich, R., Sontacci, A., Brandl, S., and Priebsch, H. H. Efficent transfer path analysis for vehicle sound engineering, Proc. JSAE Annual Congress Proceedings, Japan, (2008).

22 Özşahin, O., Özgüven, H. N., and Budak, E. Analysis and compensation of mass loading effect of accelerometers on tool point FRF measurements for chatter stability predictions, International Journal of Machine Tools and Manufacture, 50 (6), 585-589, (2010). https://dx.doi.org/10.1016/j.ijmachtools.2010.02.002

23 Cakar, O. and Sanliturk, K. Y. Elimination of transducer mass loading effects from frequency response functions, Mechanical Systems and Signal Processing, 19 (1), 87-104, (2005). https://dx.doi.org/10.1016/s0888-3270(03)00086-4

24 Bi, S., Ren, J., Wang, W., and Zong, G. Elimination of transducer mass loading effects in shaker modal testing, Mechanical Systems and Signal Processing, 38 (2), 265-275, (2013). https://dx.doi.org/10.1016/j.ymssp.2013.02.010

25 Dossing, O. Prediction of transducer mass-loading effects and identification of dynamic mass, Proc. 9th Int. Modal Analysis Conference, 6, Florence, Italy, (1991).

26 Peres, M. A., Bono, R. W., and Avitabile, P. Effects of shaker, stinger and transducer mounting on the measured frequency response functions, Proc. 6th Int. Seminar on Modal Analysis, 15, Leuven, Belgium, (2012).

27 Tönshoff, H. K. and Ahlborn, D. Sensor orientation errors in frequency response function measurements, Int. Journal of Analytical and Experimental Modal Analysis, 8 (3), 9, (1993).
28 Kim, Y. and Nelson, P. A. Optimal regularisation for acoustic source reconstruction by inverse methods, Journal of Sound and Vibration, 275 (3-5), 463-487, (2004). https://dx.doi.org/10.1016/j.jsv.2003.06.031

29 Gajdatsy, P., Janssens, K., Desmet, W., and Van der Auweraer, H. Application of the transmissibility concept in transfer path analysis, Mechanical Systems and Signal Processing, 24 (7), 1963-1976, (2010). https://dx.doi.org/10.1016/j.ymssp.2010.05.008

30 Ogden, R. W. Non-linear Elastic Deformations, Dover Publications, (1997).

31 Ogden, J. The Non-linear Properties of Rubber, BS, University of Southampton, Southampton, UK, (2010).

32 Yeoh, O. H. Some Forms of the Strain Energy Function for Rubber, Rubber Chemistry and Technology, 66 (5), 18, (1993). https://dx.doi.org/10.5254/1.3538343

33 Rivlin, R. S. Large elastic deformations of isotropic materials. IV. Further developments of the general theory, Philosophical Transactions of the Royal Society of London, Series A, Mathematical and Physical Sciences, 241, 19, (1948). https://dx.doi.org/10.1098/rsta.1948.0024

34 Heller, V. and Chaplin, J. R. Dynamic mechanical analysis of rubber used in Anaconda testing, Proc. 9th European Wave and Tidal Energy Conference, Paper 373, Southampton, UK, (2011).

35 Zhou, Z. Creep and stress relaxation of an incompressible viscoelastic material of the rate type, International Journal of Solids and Structures, 28 (5), 617-630, (1991). https://dx.doi.org/10.1016/0020-7683(91)90175-f

36 Cortés, F. and Elejabarrieta, M. J. Modelling viscoelastic materials whose storage modulus is constant with frequency, International Journal of Solids and Structures, 43 (25-26), 7721-7726, (2006). https://dx.doi.org/10.1016/j.ijsolstr.2006.03.022

37 Haneczok, G. and Weller, M. A fractional model of viscoelastic relaxation, Materials Science and Engineering: $\quad$ A, 370 (1-2), 209-212, (2004). https://dx.doi.org/10.1016/j.msea.2003.01.009

38 Roylance, D. Engineering Viscoelasticity, Massachusetts Institute of Technology, Cambridge, MA, USA, 37, (2001).

39 Wikipedia, Generalized Maxwell model, (2015).

40 Marvalova, B. Viscoelastic properties of filled rubber. Experimental observations and Material Modelling, Engineering Mechanics, 14 (1-2), 81-89, ISSN 1802-1484, (2007).

41 Haupt, P. and Sedlan, K. Viscoplasticity of elastomeric materials: experimental facts and constitutive modelling, Archive of Applied Mechanics, 71 (2-3), 89-109, (2001). https://dx.doi.org/10.1007/s004190000102

42 Huang, S. Dynamic analysis of assembled structures with nonlinearity, Ph.D., Imperial College London, London, UK, (2007). 